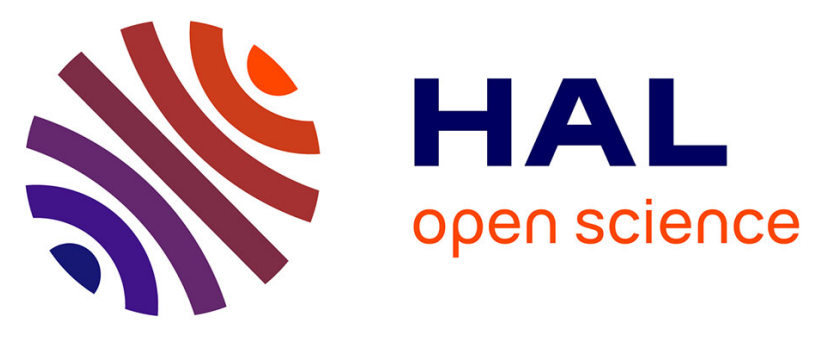

\title{
Sustained Remission After Treatment Withdrawal in Autoimmune Hepatitis: A Multicenter Retrospective Study
}

\author{
Yasmina Ben Merabet, Coralie Barbe, Alexandra Heurgue-Berlot, Thierry \\ Thévenot, Anne Minello, Francois Habersetzer, Didier Samuel, Brigitte \\ Bernard-Chabert, Delphine Weil-Verhoeven, Pascal Renard, et al.
}

\section{To cite this version:}

Yasmina Ben Merabet, Coralie Barbe, Alexandra Heurgue-Berlot, Thierry Thévenot, Anne Minello, et al.. Sustained Remission After Treatment Withdrawal in Autoimmune Hepatitis: A Multicenter Retrospective Study. Digestive Diseases and Sciences, In press, 10.1007/s10620-020-06444-7 . hal02917284

\author{
HAL Id: hal-02917284 \\ https://hal.science/hal-02917284
}

Submitted on 2 Feb 2021

HAL is a multi-disciplinary open access archive for the deposit and dissemination of scientific research documents, whether they are published or not. The documents may come from teaching and research institutions in France or abroad, or from public or private research centers.
L'archive ouverte pluridisciplinaire HAL, est destinée au dépôt et à la diffusion de documents scientifiques de niveau recherche, publiés ou non, émanant des établissements d'enseignement et de recherche français ou étrangers, des laboratoires publics ou privés. 
Sustained remission after treatment withdrawal in autoimmune hepatitis: a multicenter retrospective study

Yasmina Ben Merabet, $\mathrm{MD}^{1}$, Coralie Barbe, $\mathrm{MD}, \mathrm{PhD}^{2}$, Alexandra Heurgue-Berlot, $\mathrm{MD}^{1}$, Thierry Thévenot, $\mathrm{MD}, \mathrm{PhD}^{3}$, Anne Minello, $\mathrm{MD}, \mathrm{PhD}^{4}$, Francois Habersetzer, $\mathrm{MD}, \mathrm{PhD}^{5}$, Didier Samuel, $\mathrm{MD}, \mathrm{PhD}^{6}$, Brigitte Bernard-Chabert, $\mathrm{MD}^{1}$, Delphine Weil-Verhoeven, $\mathrm{MD}^{3}$, Pascal Renard, $\mathrm{MD}^{7}$, Hélène Clot, $\mathrm{MD}^{1}$, Vincent Di Martino,MD, $\mathrm{PhD}^{3}$, Hervé Louvet, $\mathrm{MD}^{7}$, Solange Bresson-Hadni, $\mathrm{MD}, \mathrm{PhD}^{8}$, Gérard Thiéfin, $\mathrm{MD}^{1}$.

${ }^{1}$ Department of Hepato-Gastroenterology and Digestive Oncology, Reims University Hospital, 51 092 Reims, France

${ }^{2}$ Department of Research and Public Health, Reims University Hospital, 51092 Reims, France

${ }^{3}$ Department of Hepatology, Besançon University Hospital, 25030 Besançon, France

${ }^{4}$ Department of Hepato-Gastroenterology, Dijon University Hospital, INSERM LNC-UMR 1231, University of Burgundy, 21000 Dijon, France

${ }^{5}$ Hepato-Digestive Pole, Strasbourg University Hospitals, INSERM 1110, University of Strasbourg, 67091 Strasbourg, France

${ }^{6}$ Liver Intensive Care Unit, Centre Hépato-Biliaire, AP-HP Hôpital Paul-Brousse, 94800 Villejuif, France

${ }^{7}$ Hepato-Gastroenterology private office, Reims-Bezannes Polyclinic, 51430 Bezannes, France ${ }^{8}$ Liver diseases private office, 25000 Besançon, France,

E-mails:

Yasmina Ben Merabet: yasminabenmerabet@hotmail.fr

Coralie Barbe: cbarbe@chu-reims.fr 
Alexandra Heurgue-Berlot: aheurgue @ chu-reims.fr

Thierry Thévenot: tthevenot@chu-besancon.fr

Anne Minello: anne.minello@chu-dijon.fr

Francois Habersetzer: francois.habersetzer@chru-strasbourg.fr

Didier Samuel: didier.samuel@aphp.fr

Brigitte Bernard-Chabert: bbernard-chabert@chu-reims.fr

Delphine Weil-Verhoeven: dweil@chu-besancon.fr

Pascal Renard: pascalrenard51@gmail.com

Hélène Clot: helene.clot@gmail.com

Vincent Di Martino: vdimartino@chu-besancon.fr

Hervé Louvet: hlouvetaol@ orange.fr

Solange Bresson-Hadni: dr.bresson.hadni@wanadoo.fr

Gérard Thiéfin: gthiefin@ @ chu-reims.fr

Corresponding author: Pr Gerard Thiéfin, Service d'Hépato-Gastroentérologie et de Cancérologie

Digestive, Centre Hospitalier Universitaire de Reims, 51092 Reims, France.

Phone numbrer : +336875173 44. Fax number: +33326788836

Electronic mail address: gthiefin@ chu-reims.fr

Conflict of interest: The authors declare no conflict of interest that pertain to this work

Financial support: no funding. 


\begin{abstract}
Background

In patients with autoimmune hepatitis $(\mathrm{AIH})$, relapse rates between $25 \%$ and $100 \%$ after treatment withdrawal have been reported. The optimal strategy for immunosuppressive treatment withdrawal
\end{abstract} is controversial.

Aim

To identify the predictive factors of histological remission and to assess the relapse rate after treatment withdrawal in AIH patients with prolonged biochemical response

Methods

Patients with AIH and sustained biochemical remission on first-line treatment were retrospectively included. Histological response was defined as complete regression of interface hepatitis and lobular necrosis and no or minimal portal inflammation and relapse as any elevation of serum aminotransferase or gammaglobulin/IgG levels.

Results

Sixty-two patients were included. Forty-seven had a biopsy after a median biochemical response of 49.7 months. Twenty-five of them were histological responders. Independent predictors of histological remission were older age $(\mathrm{OR}=1.1$; $\mathrm{CI95 \%}$ : 1.0;1.2), mild to moderate fibrosis at diagnosis (OR=8.; CI: 1.4;47.6) and aspartate aminotransferases < 0.6xULN (OR=7.1; CI: 1.3;36.7). Thirty-nine patients stopped therapy after a median biochemical response of 48.6 months. Twenty-four of them had a biopsy before treatment withdrawal: 21 were histological responders. The cumulative rate of relapse was $25 \%$ at 64 months.

Conclusions

This study indicates that older age, mild to moderate fibrosis at diagnosis and serum aspartate aminotransferases in the lower range of normal are independent predictors of histological response in AIH with prolonged biochemical response. The relapse rate after treatment withdrawal may be 
limited to $25 \%$ at 64 months when patients are selected on the basis of prolonged biochemical remission and, when available, histological response.

Keywords: Hepatitis, Autoimmune; Recurrence; Azathioprine; Adrenal Cortex Hormones; Liver, biopsy. 


\section{Introduction}

Autoimmune hepatitis $(\mathrm{AIH})$ is a chronic inflammatory disease of the liver due to a breakdown of immune tolerance resulting in an immune response directed against hepatocytes. If left untreated, the resulting necrotico-inflammatory activity can lead to the progressive development of cirrhosis and its complications [1,2].

The standard therapy of $\mathrm{AIH}$ is based on the combination of corticosteroids and azathioprine. Full biochemical response is defined as normalisation of both serum aminotransferase and gammaglobulin or immunoglobulin $\mathrm{G}$ (IgG) levels [1,2]. Once the response has been achieved, the recommended treatment for maintaining remission is azathioprine alone or in combination with low-dose corticosteroids according to an individual benefit-risk assessment [1-3]. In case of intolerance to azathioprine, mycophenolate mofetil is an alternative [2,4]. In case of failure, second line therapy is primarily based on tacrolimus and ciclosporine [2,4]. The optimal strategy for immunosuppressive treatment withdrawal is highly controversial as relapse rates between $25 \%$ and $100 \%$ have been reported [5]. There is no consensus on the duration of maintenance treatment. It is recommended that it be maintained for at least 2 years after a complete biochemical response has been obtained [1,2]. However, a remission period of at least 4 years before discontinuation of treatment has been reported to reduce further the risk of relapse compared to shorter treatments [6]. In addition, more drastic biochemical response criteria (aminotransferases in the lower range of normal values and $\operatorname{IgG}<12 \mathrm{~g} / \mathrm{L}$ ) have been reported to predict a lower risk of relapse [7]. Finally, histological response i.e. absence of interface hepatitis at the time of treatment withdrawal has been associated with a lower risk of relapse [8,9]. In contrast, patients with persistent inflammatory activity during treatment are at risk of disease progression and relapse after drug withdrawal $[9,10]$. 
The objectives of this study were to identify in patients with complete and prolonged biochemical response the predictive factors of histological remission and to assess the relapse rate after discontinuation of treatment.

\section{Methods}

\section{Patient population}

This multicenter retrospective study was conducted in 5 University Hospitals and 2 private practice centers from France. The medical files of consecutive patients diagnosed as having type 1 AIH between 1988 and 2017 and followed up in these centers were reviewed. Patients were included in the study if they: 1) fulfilled the revised criteria of the International Autoimmune Hepatitis Group (IAHG) for the diagnosis of definite or probable AIH [11]; 2) had a sustained biochemical remission on first-line immunosuppressive therapy as defined in AASLD and EASL guidelines [1,2] i.e. complete normalization of serum aminotransferases and $\gamma$-globulins or IgG; 3 ) had a liver biopsy following sustained biochemical remission or stopped treatment without liver biopsy according to the decision of the physician in charge of the patient. Patients with overlap syndrome associating AIH and primary biliary cholangitis (PBC) as defined by Chazouillères et al. [12] were also included.

Data collected from the medical files included: age, gender, other autoimmune diseases, identification of a triggering factor, biological tests including serum hepatic tests, autoantibodies profile and serum $\gamma$-globulin or IgG concentration at diagnosis and at treatment withdrawal, histopathological features at presentation and at the end of first line treatment if available, therapeutic modalities, time to remission and duration of biochemical remission, time to relapse after treatment withdrawal. 
Patient's records were anonymized and de-identified prior to analysis. Database was constituted in accordance with the reference methodology MR004 of the Commission Nationale de l'Informatique et des Libertés (n²206749, 13/09/2018).

\section{Liver Biopsy}

All patients underwent liver biopsy at the time of diagnosis. According to the clinician's decision, the biopsy was repeated in some patients after sustained biochemical remission to evaluate the risk of relapse in case of treatment withdrawal. Most clinicians did not stop the treatment in case of persisting interface hepatitis, even when mild or focal. Histopathological lesions were graded and staged according to the Metavir classification combining a necrotico-inflammatory activity score and a fibrosis score [13].

\section{Treatment modalities}

Patients were treated either with predniso(lo)ne alone or a combination of predniso(lo)ne and azathioprine. In one case, early digestive intolerance to azathioprine led to its replacement by mycophenolate mofetil. This was considered as a first-line treatment. Immunosuppressive treatment intensification or tapering during follow-up were individualized according to clinician's judgement.

\section{Response and relapse criteria}

Biochemical response to therapy was defined as complete normalization of serum aminotransferase and $\gamma$-globulin or IgG levels [1,2]. Histological response was defined as disappearance of histological activity (Metavir A0) whatever the residual degree of fibrosis: this means no interface hepatitis nor lobular necrosis, and no or minimal portal inflammation. Relapse was defined by any increase in serum aminotransferase or $\gamma$-globulin or IgG levels after treatment withdrawal. This definition is more stringent than that based on IAIHG criteria (reappearance of ALT elevation $>3$ 
times the ULN) but, as mentioned in EASL guidelines, AIH relapse may also present with milder elevation of aminotransferases [2]. In accordance with other investigators in recent studies [7, 14], we chose to use strict criteria to avoid overestimation of sustained remission rates after treatment withdrawal.

\section{Statistical analysis}

Qualitative variables were described as number and percentage and quantitative variables as median with range. Factors associated with histological remission were studied using univariate analysis (Wilcoxon tests, chi square tests or Fisher exact tests, as appropriate) and multivariate analysis (logistic regression with stepwise selection, with an exit threshold of 0.05 and factors significant at $\mathrm{p}=0.05$ included). The relapse-free survival curves were established by the Kaplan-Meier method. Variables associated with relapse after treatment withdrawal were identified by univariate analysis using Log rank tests. Given the small number of relapses, multivariate analysis was not performed. Significance level for two-tailed $p$-values was set at 0.05 . All analyses were performed using SAS version 9.4 (SAS Institute Inc., Cary, NC, USA).

\section{Results}

\section{Patient characteristics}

A total of 302 patients were identified from databases. Two hundred and forty were excluded from the study because of missing data due to lack of regular follow-up in participating centers $(n=60)$, no response to first line treatment $(n=58)$, responders still on first line treatment without second liver biopsy $(\mathrm{n}=107)$, type 2 HAI $(\mathrm{n}=5)$, no biopsy at diagnosis $(\mathrm{n}=4)$, absence of treatment $(\mathrm{n}=6)$ (Fig. 1). 
Table 1 shows the baseline characteristics of the study population at diagnosis. Associated autoimmune diseases were coeliac disease $(n=1)$, Gougerot-Sjögren syndrome $(n=3)$, Crohn's disease $(n=1)$, ulcerative colitis $(n=1)$, Biermer's disease $(n=2)$, rheumatoid arthritis $(n=2)$, lupus $(n=2)$, autoimmune thyroiditis $(n=4)$, immune thrombocytopenic purpura $(n=1)$, autoimmune hemolytic anemia $(n=1)$, vitiligo $(n=1)$.

Serum IgG level was not routinely monitored in all patients, especially prior 2010 when gammaglobulin dosage was more commonly used for the diagnosis and the follow-up of AIH patients. For this reason, we used normalization of IgG or gammaglobulins for the definition of biochemical remission. In 23 patients, both measurements were performed on the same day at diagnosis or during follow-up. As previously reported [7], serum gammaglobulin and IgG levels were tightly correlated $(\mathrm{r}=0.88, \mathrm{p}<0.0001)$ (supplementary Fig. 1)

As induction treatment, 57 patients $(91.9 \%)$ received a combination of corticosteroids and azathioprine, $4(6.5 \%)$ corticosteroids alone and $1(1.6 \%)$ a combination of corticosteroids and mycophenolate mofetil. As maintenance treatment, 51 (82.2\%) patients were treated with azathioprine monotherapy, $6(9.7 \%)$ with a combination of corticosteroids and azathioprine, 4 $(6.5 \%)$ with corticosteroid monotherapy and $1(1.6 \%)$ with mycophenolate mofetil monotherapy. The median duration of treatment was 66.6 months ((21.3-268.9). All patients but 5 had a sustained biochemical remission for at least 24 months before follow-up biopsy or treatment withdrawal (median: 48.6 months, range 10-157.8). The median follow-up was 137.4 months (range 37-359.1).

\section{Histological remission}

Forty seven patients had a follow-up biopsy after a median treatment of 66.5 months (range 21.3268.9) and a median biochemical remission of 49.7 months (range 10-151.7). Twenty five patients (53\%) were histological responders and 22 patients (47\%) had a persistent histological activity. Comparison between patients with histological remission and those with persisting histological 
activity is presented in Table 2. In univariate analysis, histological responders were significantly older than patients with persisting activity (median age: 49 versus $36, \mathrm{p}=0.007$ ), had more often mild to moderate fibrosis at diagnosis $(72 \%$ versus $36.3 \%, \mathrm{p}=0.01)$ and lower serum level of AST at the time of treatment withdrawal (58.3\% with AST < $0.6 \mathrm{ULN}$ versus $23.8 \%, \mathrm{p}=0.02)$. Seventythree percent of patients with AST < 0.6 x ULN were histological responders compared with $38.5 \%$ of those with AST in the normal range but above $0.6 \times$ ULN ( $\mathrm{p}=0.02$ ) (Fig. 2). In addition, in the subgroup of 25 patients where the dosage was available, serum $\gamma$-globulin level at treatment withdrawal was significantly lower in histological responders $(0.7$ ULN versus 0.9 ULN, p=0.03, Table 2) and the percentage of histological responders in patients with gammaglobulin level below $0.8 \times$ ULN was significantly higher than in patients with gammaglobulins in the normal range but above 0.8 x ULN (78.3\% versus 36.3\%, p=0.049) (supplementary Fig. 3). However, given the large number of missing data $(\mathrm{n}=22)$, this variable was not entered in the multivariate model. Mutivariate analysis showed that older age, mild to moderate fibrosis at diagnosis and serum AST level below 0.6 ULN at the time of follow-up biopsy were independent predictors of histological response (OR $=1.1[1.02 ; 1.18], \mathrm{p}=0.009 ; \mathrm{OR}=8.1 \quad[1.39 ; 47.6], \mathrm{p}=0.02 ; \quad \mathrm{OR}=7.05 \quad[1.35 ; 36.7], \mathrm{p}=0.02$, respectively) (Table 2).

\section{Relapse after treatment withdrawal}

Thirty nine patients with a prolonged biochemical remission discontinued the treatment after a median treatment of 61.4 months (range 25.3-268.9) and a median biochemical remission of 48.6 months (range 20.3-157.8). As shown in Fig. 3, the probability of relapse was $12 \%$ at 12 months and $25 \%$ at 64 months. The median time of relapse was 9.4 months (range 1.5-64).

Twenty four of these patients $(61.5 \%)$ had a liver biopsy before the treatment withdrawal. The great majority of them, 21 out 24, were histological responders. Among them, two relapsed at 2 and 
10 months after treatment withdrawal. Three patients (8\%) had persisting activity, among which 2 relapsed at 14 and 16 months.

Among the 5 patients with an identifiable trigger factor (hepatitis virus A, cytomegalovirus infection, furadantine and minocycline in 2 patients), 4 stopped their treatment and did not relapse and one (AIH triggered by hepatitis virus A ) had still mild histological activity at a follow-up biopsy and was maintained on treatment, then lost of follow-up. The cumulative rate of relapse was re-analyzed after exclusion of these patients. It was $13.4 \%$ at 12 months and $28.9 \%$ at 64 months. The median time of relapse was 9.4 months (range1.5-64) (supplementary Fig. 3).

\section{Patient characteristics associated with the risk of relapse}

Comparison between patients with sustained remission and relapsers is presented in Table 3. No significant difference was observed for age at diagnosis, sex ratio, presence of other autoimmune diseases, severity of the disease at diagnosis and serum $\gamma$-globulin or IgG concentrations at diagnosis and treatment withdrawal. The only significant difference between the two groups was a higher prevalence of anti-smooth muscle antibodies at diagnosis in relapsers than in sustained responders $(100 \%$ versus $50 \%, \mathrm{p}=0.005)$. Of note, azathioprine treatment tended to be longer in sustained responders than in relapsers (62.7 months versus 44.2 months, $\mathrm{p}=0.055)$.

In the subgroup of 24 patients who had a liver biopsy before treatment withdrawal, most of them were histological responders as clinicians usually did not stop the treatment in case of persisting activity at the follow-up biopsy.

\section{Evolution of fibrosis}

In the group of 47 patients who had a follow-up biopsy after a median treatment of 66.5 months (range $21.3 ; 268.9)$, the degree of fibrosis decreased in 26 patients $(55 \%)$, was stable in 20 patients 
$(43 \%)$ and worsened in only one patient $(2 \%)$. Globally, there was a significant improvement in the level of fibrosis $(\mathrm{p}=0.007)$ (Table 4).

\section{Discussion}

Our study analyzed a series of $62 \mathrm{AIH}$ patients with prolonged biochemical remission i.e. complete normalization of serum aminotransferases and $\gamma$-globulins or IgG [1]. Forty seven of these patients (53\%) underwent liver biopsy after a median of 48 months of biochemical response. Fifty-three per cent of them had a complete histological response defined as complete disappearance of interface hepatitis or lobular necrosis, even mild or focal, indicating that complete biochemical response even prolonged does not preclude the persistence of histological activity in a significant number of patients. Independent predictors of histological remission were older age, mild to moderate hepatic fibrosis at diagnosis and serum AST levels in the lower range of normal at the time of follow-up biopsy. In addition, serum gammaglobulins in the lower range of normal were also significantly associated with histological response in univariate analysis. This suggests that more stringent criteria of biochemical response should be used to better predict histological response. In 39 patients stopping treatment after a median of 48.6 months of biochemical response, 24 of them after follow-up biopsy, the relapse rate was $25 \%$ at 64 months.

It is well-established that increased levels of serum aminotransferases and/or gammaglobulins/IgG in AIH patients reliably predict ongoing histological activity [10, 15]. However, the predictive value of normalization of these makers for histological response is controversial. In some studies $[10,15,16]$, rates of persisting activity between 45 and $67 \%$ have been reported whereas in Hartl et al's studies [7, 17], it was less than $20 \%$. The variability may be explained by differences in the severity of the disease, the type of treatment, the duration of biochemical remission, the low number of patients in some studies and the criteria of histological response. In our study, the rate of histological remission 
was $53 \%$ in a series of 47 biochemical responders. Whether the use of gammaglobulins or globulins $[15,16$, our study] instead of $\operatorname{IgG}$ for the definition of biochemical remission may explain the difference with Hartl et al's studies [7, 17] is uncertain. Given the limited numbers of patients in Hartl et al's studies [7, 17], the conclusion that biochemical response based on aminotransferase and IgG normalization is a surrogate marker of histological response needs to be validated in larger series. Our multivariate analysis showed that serum AST level in the lower range of normal values at the time of follow-up biopsy was an independent predictor of histological remission. In the subgroup of patients with AST levels below 0.6 x ULN, the rate of histological response significantly raised to $74 \%$ whereas in those with AST in normal range but above $0.6 \times$ ULN, the rate of histological response was $38 \%$. This is in accordance with Dhaliwal et al [16] who reported significantly lower serum aminotransferase levels in histological responders. The same tendency was also reported by Hartl et al [17] although it was not significant. Other independent predictors of histological response were older age and mild to moderate fibrosis at diagnosis. Previous studies have reported that older age was predictive of biochemical response $[18,19]$. Contradictory results have been reported regarding the prognostic impact of cirrhosis on biochemical response [18,20, 21]. In addition, we showed in univariate analysis that serum $\gamma$-globulin level at treatment withdrawal was also significantly lower in histological responders.

The utility of a follow-up liver biopsy prior to treatment withdrawal is controversial $[1,2,16,17$, 22]. The recommendation that follow-up biopsy is not warranted in patients with prolonged biochemical remission is based on the analysis of a limited number of patients [17]. Like others [16], we think, based on our data, that a follow-up biopsy should always be discussed as persisting histological activity justifies an extension of immunosuppressive treatment. However, in accordance with guidelines $[1,2,22]$, we agree that it may not be mandatory in all patients and should be individualized. In reluctant patients, treatment withdrawal without biopsy may be an option, especially in those who have favourable predictive factors such as advanced age, no or minimal 
fibrosis at diagnosis and aminostransferases and gammaglobulins/IgG in the lower range of normal under maintenance treatment. In every case, close clinical and biochemical monitoring should be maintained after treatment withdrawal to detect a relapse at an early stage. Reinstitution of the same immunosuppressive regimen is usuallly efficient to reinduce complete remission $[1,2,22]$. In our series of 39 patients with prolonged biochemical response, the rate of relapse was $25 \%$ at 64 months, lower than in most previous studies. In those using loose criteria for the definition of biochemical remission i.e. aminotransferases below 2 ULN, relapse after treatment withdrawal was reported to occur in as many as $50 \%$ of the patients within 6 months after discontinuation of therapy [23] and around $75 \%$ of the patients who had a longer follow up [24-26]. Even with complete normalisation of aminotransferase before treatment withdrawal, relapse rate between 55 and $100 \%$ were still reported [6,27-30]. Inclusion of paediatric cases, various regimens, various definitions and durations of biochemical remission and various definitions of relapse may explain differences between studies. In a large multicenter Dutch study [27], $131 \mathrm{HAI}$ patients with normalization of serum ALT levels for at least 2 years were included. The rate of relapse after stopping treatment was $81 \%$ at three years. However, in approximately $50 \%$ of these patients, no information was available on serum IgG level at the time of drug withdrawal. In addition, $24 \%$ of the patients were treated with a second-line therapy after a first relapse, a condition known to increase the risk of relapse compared with first line treated patients. Fifty-four $\%$ of the patients were still on corticosteroids combined with azathioprine at the time of treatment withdrawal. Finally, $21 \%$ were under 18 years of age at the time of diagnosis, a condition known to be associated with a higher rate of relapse [1]. In contrast, using the current definition of biochemical remission, i.e. complete normalisation of aminotransferases and IgG for at least 2 years, Hartl et al [7] reported a relapse rate of $46 \%$ after a median follow-up of 28 months in a series of 28 patients and Zachou et al. [14] a relapse rate of $25 \%$ after a median follow-up of 24 months in a series of 40 patients. In addition, Kanzler et al [6] reported that the relapse rate was dependent on the duration of immunosuppressive 
therapy. In a series of 28 patients with complete biochemical remission, the subgroup of those with more than 4 years of continuous immunosuppressive therapy prior to treatment withdrawal had a probability of relapse of only $33 \%$ compared with $83 \%$ in the subgroup of patients treated for $2-4$ years. In our study, the use of strict criteria for biochemical and histological remission and the long duration of biochemical remission may explain the low rate of relapse. In addition, most patients who had a follow-up biopsy before treatment withdrawal were also histological responders (21 out of 24. In accordance with current recommendations which discourage treatment discontinuation in case of interface hepatitis on the follow-up biopsy [2], very few patients with persisting histological activity had their treatment withdrawn in our series. Only 3 such patients stopped their treatment and 2 of them relapsed 14 and 15 months after treatment withdrawal. Besides, our study confirms that the lack of histological activity on the follow-up biopsy is predictive of a high rate of sustained response, near to $90 \%$ at 5 years in our study. Finally, as the inclusion of AIH cases with a triggering factor in our series may have led to underestimate the risk of relapse [31], the cumulative rate of relapse was re-analyzed after exclusion of these cases. It was still low at $28.9 \%$ at 64 months.

Analysis of the factors associated with relapse was limited due the small number of relapses in our population (7 out of 37). Results should be interpreted cautiously. Univariate analysis showed that the prevalence of ASMA at diagnosis was significantly higher in relapsers than in sustained responders. Contradictory results have been published regarding the predictive value of ASMA in HAI patients. Couto et al [32] have reported that the persistence of ASMA or anti-actin antibodies (AAA) during treatment was significantly associated with persistent biochemical and histological activity. However, in this study, the reactivity of ASMA or AAA at diagnosis, before treatment institution, was not associated with response to treatment [32]. In contrast, in a series of $282 \mathrm{AIH}$ patients, Muratori et al [18] have reported a higher frequency of seropositivity for ASMA in biochemical non responders than in responders. In addition, Czaja et al [33] have suggested the 
prognostic value of AAA seropositivity at diagnosis, with a higher rate of transplantation and death in HAI patients positive for AAA. In our study, the prevalence of ASMA was available at diagnosis but not at treatment withdrawal and AAA were tested only in a small number of patients. Our findings should be considered as exploratory and need to be interpreted carefully.

The low rate of relapse and the number of missing data were limiting factors for identification of other predictive factors in our study. In a series of 28 patients, Hartl et al [7] have reported that serum ALT levels below half the upper limit of normal and $\mathrm{IgG}$ levels below $12 \mathrm{~g} / \mathrm{L}$ were predictive of sustained response after treatment withdrawal. It is of interest to underline that, in our study, low serum levels of AST below $0.6 \mathrm{~N}$ et gammaglobulins in lower range of normal were predictive of histological response, which is consistent with Hartl et al' results as histological remission is predictive of sustained remission.

Finally, our study confirms that immunosuppressive treatment of AIH not only prevents further progression of the disease in biochemical responders but allows significant regression of fibrosis even in cirrhotic patients. This is in accordance with the conclusions of a recent study reporting that complete biochemical remission was the only independent predictor for histological fibrosis regression especially in patients with extensive fibrosis or cirrhosis [17]. In addition, it has been shown that patients who experienced laboratory and histological resolution of inflammatory manifestations within 12 months of therapy had lower frequency of progression to cirrhosis and lower frequency of death from hepatic failure or need for liver transplantation [19].

Our study has some limitations. It is a retrospective study with inherent limitations such as missing data, patients lost of follow-up and treatment bias. The number of patients in our study was relatively low but similar to series reported in recent studies using the same stringent inclusion criteria $[6,7,14]$ as discussed above. The number of relapses after treatment withdrawal was too low in our series to allow identification of predictive factors. However, we were able to identify predictors of complete histological remission, a condition associated with a low relapse rate. 
In conclusion, our study did not confirm the recent suggestion that prolonged biochemical response in AIH patients may be used as a surrogate marker of histological response [17]. Although not mandatory, follow-up biopsy still appears of clinical interest to identify patients with persisting histological activity who are prone to relapse in case of treatment withdrawal. When patients are selected on the basis of prolonged biochemical remission and, when available, histological response, the relapse rate after treatment withdrawal may be limited to $25 \%$ at 64 months.

\section{References}

1. Manns MP, Czaja AJ, Gorham JD, Krawitt EL, Mieli-Vergani G, Vergani D; American Association for the Study of Liver Diseases. Diagnosis and management of autoimmune hepatitis. Hepatology, 2010;51:2193-2213.

2. European Association for the Study of the Liver. EASL Clinical Practice Guidelines: Autoimmune hepatitis. J Hepatol, 2015;63:971-1004.

3. Mieli-Vergani G, Vergani D, Czaja AJ, et al. Autoimmune hepatitis. Nat Rev Dis Primers, 2018;4:18017.

4. Efe C, Hagström H, Ytting H, et al. Efficacy and Safety of Mycophenolate Mofetil and Tacrolimus as Second-line Therapy for Patients With Autoimmune Hepatitis. Clin Gastroenterol Hepatol, 2017;15:1950-1956.

5. Harrison L, Gleeson D. Stopping immunosuppressive treatment in autoimmune hepatitis (AIH): Is it justified (and in whom and when)? Liver Int, 2019;39:610-620.

6. Kanzler S, Gerken G, Löhr H, Galle PR, Meyer zum Büschenfelde KH, Lohse AW. Duration of immunosuppressive therapy in autoimmune hepatitis. J Hepatol, 2001;34:354-355.

7. Hartl J, Ehlken H, Weiler-Normann C, et al. Patient selection based on treatment duration and liver biochemistry increases success rates after treatment withdrawal in autoimmune hepatitis. $J$ Hepatol, 2015;62:642-646. 
8. Czaja AJ, Davis GL, Ludwig J, Taswell HF. Complete resolution of inflammatory activity following corticosteroid treatment of HBsAg-negative chronic active hepatitis. Hepatology, 1984;4:622-627.

9. Czaja AJ. Review article: the management of autoimmune hepatitis beyond consensus guidelines. Aliment Pharmacol Ther, 2013;38:343-364.

10. Lüth S, Herkel J, Kanzler S, et al. Serologic markers compared with liver biopsy for monitoring disease activity in autoimmune hepatitis. J Clin Gastroenterol, 2008;42:926-930.

11. Alvarez F, Berg PA, Bianchi FB, et al. International Autoimmune Hepatitis Group Report: review of criteria for diagnosis of autoimmune hepatitis. J Hepatol, 1999;31:929-938.

12. Chazouillères $\mathrm{O}$, Wendum $\mathrm{D}$, Serfaty L, et al. Primary biliary cirrhosis-autoimmune hepatitis overlap syndrome: clinical features and response to therapy. Hepatology, 1998;28:296-301.

13. Bedossa P, Poynard T. An algorithm for the grading of activity in chronic hepatitis C. The METAVIR Cooperative Study Group. Hepatology, 1996;24:289-293.

14. Zachou K, Gatselis NK, Arvaniti P, et al. A real-world study focused on the long-term efficacy of mycophenolate mofetil as first-line treatment of autoimmune hepatitis. Aliment Pharmacol Ther, 2016;43:1035-1047.

15. Czaja AJ, Wolf AM, Baggenstoss AH. Laboratory assessment of severe chronic active liver disease during and after corticosteroid therapy: correlation of serum transaminase and gamma globulin levels with histologic features. Gastroenterology, 1981;80:687-692.

16. Dhaliwal HK, Hoeroldt BS, Dube AK, et al. Long-term prognostic significance of persisting histological activity despite biochemical remission in autoimmune hepatitis. $A m \quad J$ Gastroenterol, 2015;110:993-999.

17. Hartl J, Ehlken H, Sebode M, et al. Usefulness of biochemical remission and transient elastography in monitoring disease course in autoimmune hepatitis. J Hepatol, 2018;68:754763. 
18. Muratori P, Lalanne C, Bianchi G, Lenzi M, Muratori L. Predictive factors of poor response to therapy in Autoimmune Hepatitis. Dig Liver Dis, 2016;48:1078-1081.

19. Czaja AJ. Rapidity of treatment response and outcome in type 1 autoimmune hepatitis. $J$ Hepatol, 2009;51:161-167.

20. Ngu JH, Gearry RB, Frampton CM, Stedman CA. Predictors of poor outcome in patients with autoimmune hepatitis: a population-based study. Hepatology, 2013;57:2399-2406.

21. Roberts SK, Therneau TM, Czaja AJ. Prognosis of histological cirrhosis in type 1 autoimmune hepatitis. Gastroenterology, 1996;110:848-857.

22. Gleeson D, Heneghan MA; British Society of Gastroenterology. British Society of Gastroenterology (BSG) Guidelines for Management of Autoimmune Hepatitis British Society of Gastroenterology (BSG) guidelines for management of autoimmune hepatitis. Gut, 2011;60:1611-1629.

23. Czaja AJ, Ammon HV, Summerskill WHJ. Clinical features and prognosis of severe chronic active liver disease (CALD) after corticosteroid-induced remission. Gastroenterology, $1980 ; 78: 518-523$

24. Czaja AJ, Ludwig J, Baggenstoss AH, et al. Corticosteroid-treated chronic active hepatitis in remission: uncertain prognosis of chronic persistent hepatitis. N Engl J Med, 1981;304:5-9.

25. Verma S, Gunuwan B, Mendler, M, Govindrajan S, Redeker A. Factors predicting relapse and poor outcome in type I autoimmune hepatitis: role of cirrhosis development, patterns of transaminases during remission and plasma cell activity in the liver biopsy. Am $J$ Gastroenterol, 2004;99:1510-1516.

26. Montano-Loza AJ, Carpenter HA, Czaja AJ. Improving the end point of corticosteroid therapy in type 1 autoimmune hepatitis to reduce the frequency of relapse. Am J Gastroenterol, 2007;102:1005-1012. 
27. Hegarty JE, Nouri Aria KT, Portmann B, Eddleston AL, Williams R. Relapse following treatment withdrawal in patients with autoimmune chronic active hepatitis. Hepatology, $1983 ; 3: 685-689$.

28. van Gerven NMF, Verwer BJ, Witte BI, van Hoek B, Coenraad MJ, van Erpecum KJ. Relapse is almost universal after withdrawal of immunosuppressive medication in patients with autoimmune hepatitis in remission. J Hepatol, 2013;58:141-147.

29. Kirstein MM, Metzler F, Geiger E, et al. Prediction of short- and long-term outcome in patients with autoimmune hepatitis. Hepatology. 2015;62:1524-1535.

30. Muratori P, Granito A, Quarneti C, et al. Autoimmune hepatitis in Italy: the Bologna experience. J Hepatol, 2009;50:1210-1218.

31. Bjornsson E, Talwalkar J, Treeprasertsuk S, Kamath PS, Takahashi N, Sanderson S, et al. Drug-induced autoimmune hepatitis: clinical characteristics and prognosis. Hepatology, 2010;51:2040-2048.

32. Couto CA, Bittencourt PL, Porta G, et al. Antismooth muscle and antiactin antibodies are indirect markers of histological and biochemical activity of autoimmune hepatitis. Hepatology, 2014;59:592-600.

33. Czaja AJ, Cassani F, Cataleta M, et al. Frequency and significance of antibodies to actin in type 1 autoimmune hepatitis. Hepatology, 1996;24:1068-1073. 
Fig. 1 Flow chart of the study.

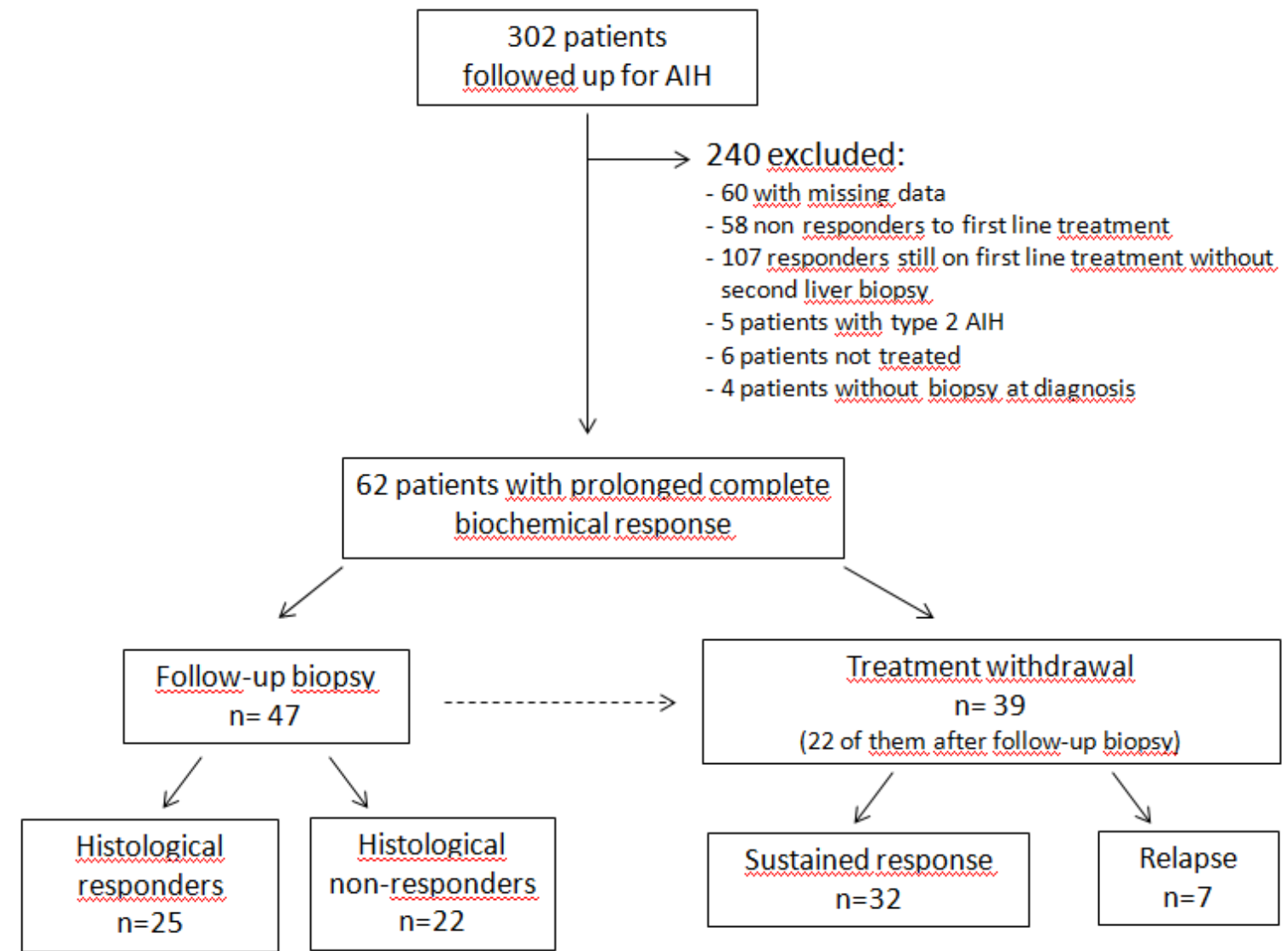


Fig. 2 Serum ASAT concentration in histological responders and non-responders after a median biochemical response of 48.8 and 51.8 months respectively. A. Median and interquartile range ( $\mathrm{p}=0.02$, Wilcoxon test). B. Percentages of histological responders in patients with ASAT $<0.6 \mathrm{x}$ ULN and those with ASAT in the normal range but above $0.6 \times$ ULN ( $<<0.02$, chi square test). ULN: upper level of normal, Missing data=2

A.
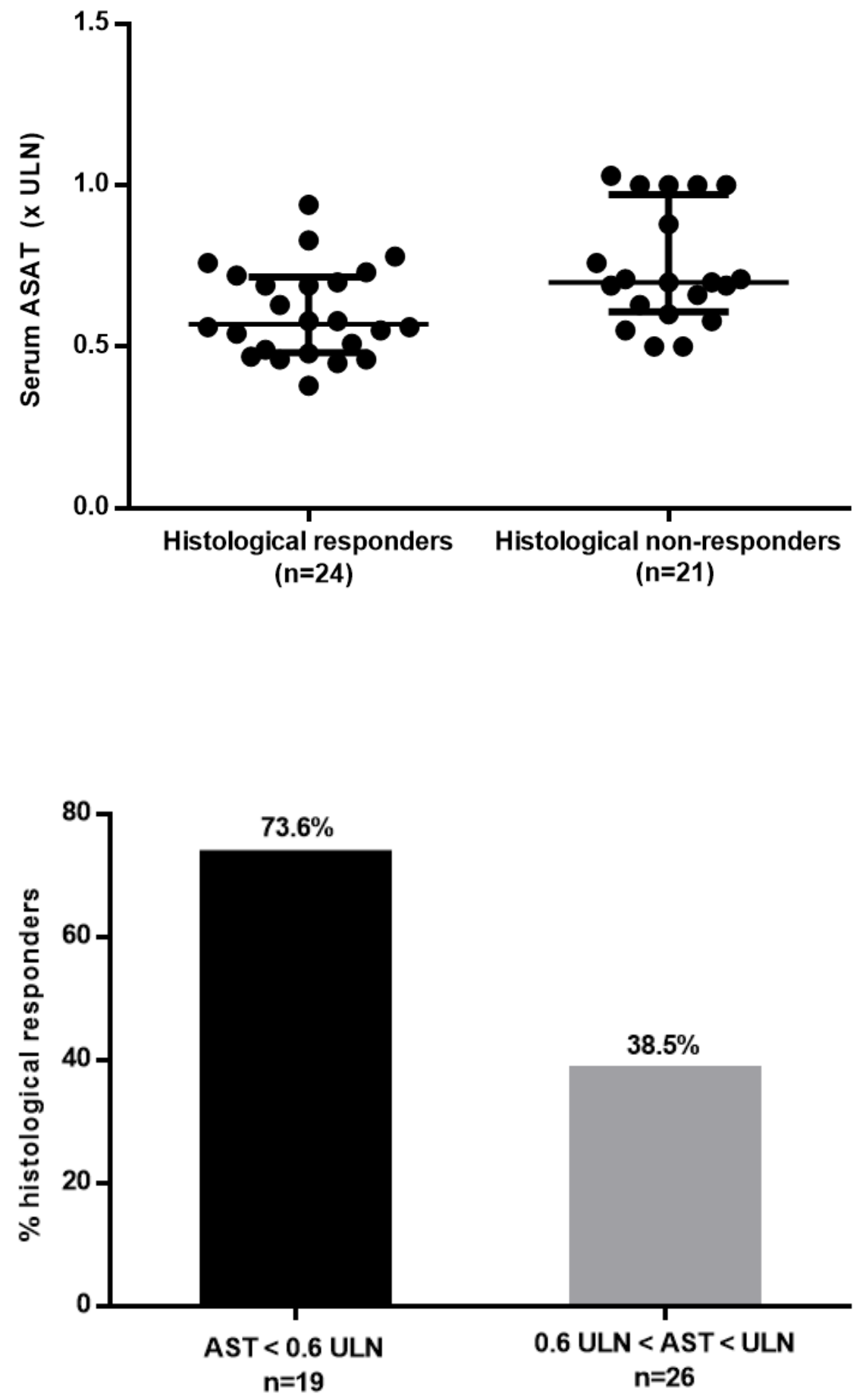
Fig. 3 Kaplan Meier plot: Probability of relapse after drug withdrawal in 39 AIH patients with biochemical remission for a median time of 48 months.

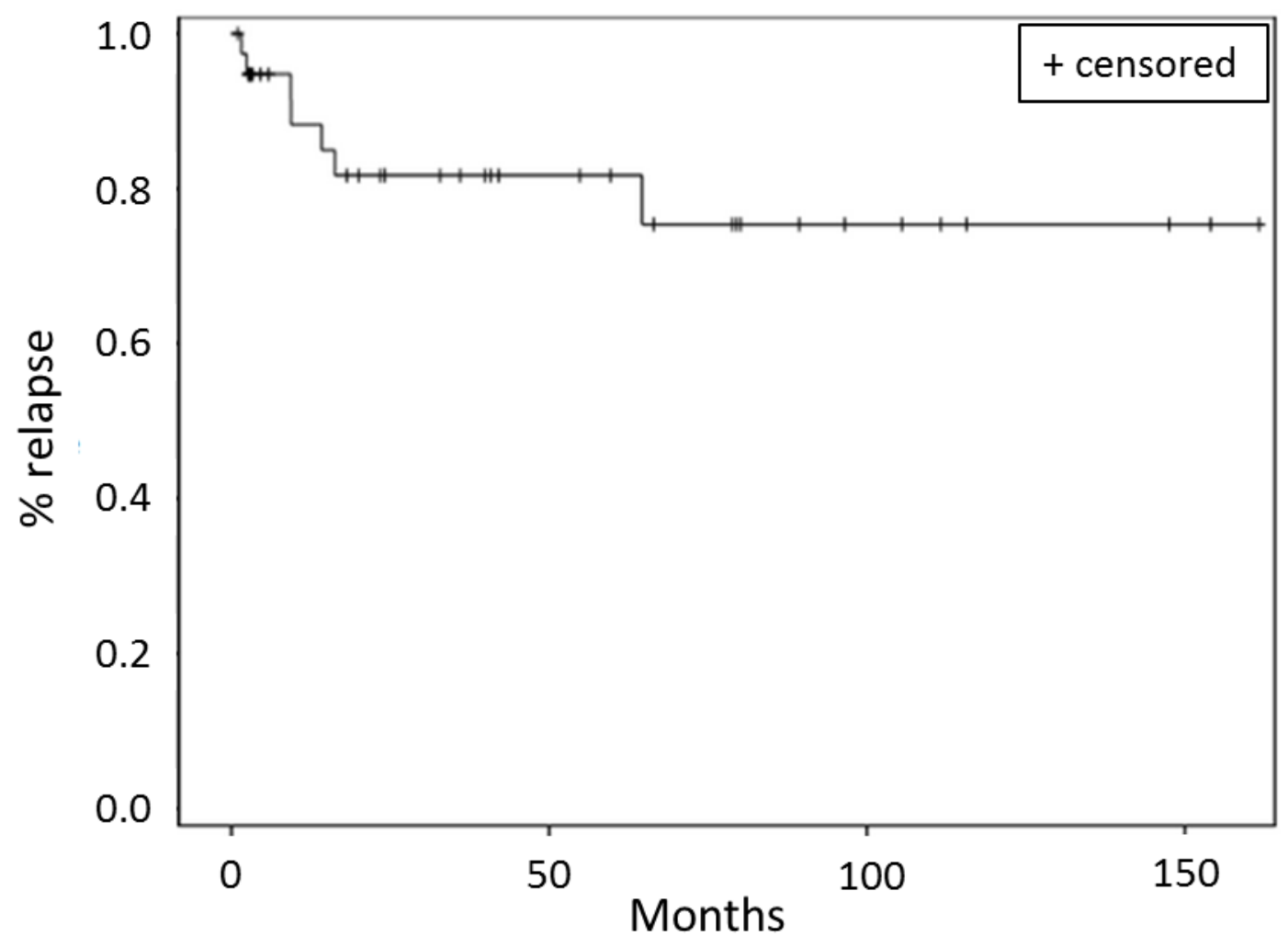


Table 1. Baseline data in $62 \mathrm{AIH}$ patients.

\begin{tabular}{|c|c|}
\hline Variables & \\
\hline Age at diagnosis, years, median (range) & $44(11-73)$ \\
\hline Sex ratio, Females/males & $47 / 15(76 / 24)$ \\
\hline Trigger factors* & $5(8.1)$ \\
\hline Other auto-immune diseases & $17(27.4)$ \\
\hline Overlap syndrome HAI-CBP & $5(8.1)$ \\
\hline Score IAGH, probable/certain & $17 / 45(27 / 73)$ \\
\hline 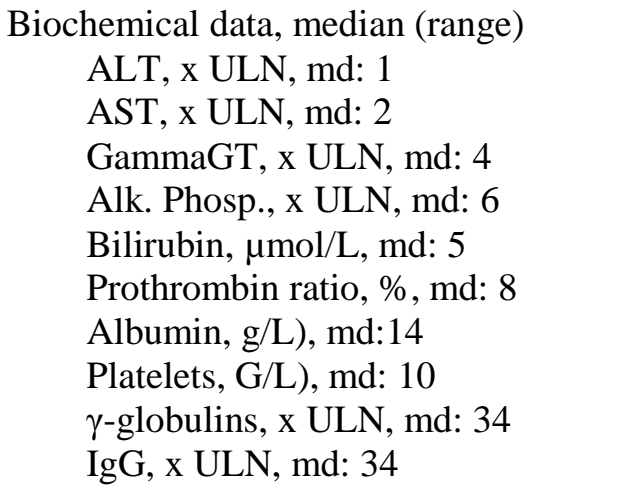 & $\begin{array}{l}12.1(1.4-81) \\
7.9(1.1-56.3) \\
4.6(1-21.5) \\
1.3(1-6.3) \\
28(4-467) \\
87.5(23-100) \\
39(22-50) \\
254(109-545) \\
1.2(0.8,1.9) \\
1.4(0.5-2.2)\end{array}$ \\
\hline $\begin{array}{l}\text { Auto-antibodies } \\
\text { ANA } \\
\text { ASMA md:1 } \\
\text { Anti-LKM md: } 8 \\
\text { AMA md:2 } \\
\text { Anti-SLA md: } 50 \\
\text { Anti-LC1 md: } 24\end{array}$ & $\begin{array}{l}50(80.6) \\
35(56.4) \\
1(1.8) \\
5(8.3) \\
2(16.7) \\
0\end{array}$ \\
\hline $\begin{array}{c}\text { METAVIR A } \\
\text { A0 } \\
\text { A1 } \\
\text { A2 } \\
\text { A3 }\end{array}$ & $\begin{array}{l}0(0.0) \\
9(14.5) \\
19(30.7) \\
34(54.8)\end{array}$ \\
\hline $\begin{array}{c}\text { METAVIR F } \\
\text { F0 } \\
\text { F1 } \\
\text { F2 } \\
\text { F3 } \\
\text { F4 }\end{array}$ & $\begin{array}{l}3(4.8) \\
13(21) \\
21(33.9) \\
15(24.2) \\
10(16.1)\end{array}$ \\
\hline
\end{tabular}

Data are expressed as No. (\%) unless otherwise indicated. Continuous variables are reported as median (range). ALT: alanine aminotransferase; AST: aspartate aminotransferase; Alk. Phosph.: alkaline phosphatase; ANA: antinuclear antibody; ASMA: antismooth muscle antibody; AMA: antimitochondrial antibody; LKM: liver/kidney microsomal; ULN: upper limit of normal; md: missing data; A:activity; F: fibrosis liver biopsy 1: at diagnosis; liver biopsy 2: at the end of first line treatment. *virus A $(n=1)$, CMV $(n=1)$ minocycline $(n=2)$, furadantine $(n=1)$ 
Table 2. Comparisons of Demographics, clinical, laboratory and histopathological data and treatment characteristics between patients with and without histological response

\begin{tabular}{|c|c|c|c|c|c|c|}
\hline & \multirow{2}{*}{$\begin{array}{c}\text { Metavir A0 group } \\
n=25\end{array}$} & \multirow{2}{*}{$\begin{array}{c}\text { Metavir } A \geq 1 \text { group } \\
\mathrm{n}=22\end{array}$} & \multirow[t]{2}{*}{$\mathrm{p}$ value } & \multicolumn{3}{|c|}{ Multivariate analysis } \\
\hline & & & & OR & $(\mathrm{IC} 95 \%)$ & $\mathrm{p}$ \\
\hline Age at diagnosis (years) & $49(20-64)$ & $36(11-69)$ & 0.007 & 1.11 & {$[1.03 ; 1.19]$} & 0.007 \\
\hline Sex ratio (Females/males) & $19 / 6(76 / 24)$ & $16 / 6(72 / 28)$ & 0.80 & & & \\
\hline Other auto-immune diseases & $8(32)$ & $6(27)$ & 0.72 & & & \\
\hline $\begin{array}{l}\text { Biochemical data at diagnosis } \\
\text { ALT, x ULN } \\
\text { AST, x ULN, md:1 } \\
\gamma \text {-globulins, x ULN, md:22 } \\
\text { IgG, x ULN md:28 }\end{array}$ & $\begin{array}{l}12.1(2-81) \\
8.3(1.1-46) \\
1.1(0.8-1.9) \\
1.7(0.5-2.1)\end{array}$ & $\begin{array}{l}6(1.5-38.6) \\
4.5(1.2-39.6) \\
1.1(0.8-1.7) \\
1.2(0.9-2.1)\end{array}$ & $\begin{array}{l}0.21 \\
0.66 \\
0.79 \\
0.77\end{array}$ & & & \\
\hline $\begin{array}{l}\text { ANA } \\
\text { ASMA }\end{array}$ & $\begin{array}{l}22(88) \\
13(52)\end{array}$ & $\begin{array}{l}16(72.7) \\
12(27.8)\end{array}$ & $\begin{array}{l}0.27 \\
0.86\end{array}$ & & & \\
\hline $\begin{array}{l}\text { METAVIR A (Liver biopsy 1) } \\
\text { A1 } \\
\text { A2 } \\
\text { A3 }\end{array}$ & $\begin{array}{l}5(20) \\
5(20) \\
15(60)\end{array}$ & $\begin{array}{l}2(9) \\
7(32) \\
13(59)\end{array}$ & 0.51 & & & \\
\hline $\begin{array}{l}\text { METAVIR F (Liver biopsy 1) } \\
<\text { F3 } \\
\geq \text { F3 }\end{array}$ & $\begin{array}{l}18(72) \\
7(28)\end{array}$ & $\begin{array}{l}8(36.3) \\
14(73.7)\end{array}$ & 0.01 & 8.1 & {$[1.39 ; 47.6]$} & 0.02 \\
\hline $\begin{array}{c}\text { Biochemical data at Liver biopsy } 2 \\
\text { ALT, x ULN, md :1 } \\
\text { AST < 0.6 x ULN md:2 } \\
\text { Yes } \\
\text { No } \\
\text {-globulins, x ULN, md: } 22 \\
\text { IgG, x ULN, md: } 22\end{array}$ & $\begin{array}{l}0.45(0.2-1) \\
14(58.3) \\
10(41.7) \\
0.7(0.6-1) \\
0.7(0.6-1.1)\end{array}$ & $\begin{array}{l}0.5(0.1-1) \\
5(23.8) \\
16(76.2) \\
0.9(0.6-1) \\
0.8(0.5-1)\end{array}$ & $\begin{array}{l}0.39 \\
\mathbf{0 . 0 2} \\
\\
\\
\mathbf{0 . 0 3} \\
0.67\end{array}$ & 7.05 & $(1.35 ; 36.7)$ & 0.02 \\
\hline $\begin{array}{l}\text { Type of treatment } \\
\text { CT monotherapy } \\
\text { CT/AZT bitherapy }\end{array}$ & $\begin{array}{l}2(8) \\
23(92)\end{array}$ & $\begin{array}{l}0(0) \\
22(100)\end{array}$ & 0.49 & & & \\
\hline Delay to transaminases normalization, $\mathrm{md}: 1^{*}$ & $4.3(0.5-44)$ & $7.8(0.5-49.4)$ & 0.37 & & & \\
\hline
\end{tabular}


Delay to biochemical remission, md: $6^{*}$

Duration of biochemical remission, md: $6^{*}$

Duration of CT treatment*

Duration of AZT/MMF** treatment*

Duration of treatment* aspartate aminotransferase; Alk. Phosph.: alkaline phosphatase; ANA: antinuclear antibody; ASMA: anti-smooth muscle antibody; antiLKM: anti-

liver/kidney microsomal antibody; ULN: upper limit of normal; md: missing data; A:activity; F: fibrosis; AZT : azathioprine, MMF: mycophenolate mofetil.

$*$ duration in months ** 44 patients on AZT and 1 on MMF (two other patients were on corticosteroid monotherapy).

$\begin{array}{lll}7.1(0.9-44) & 10.9(0.5-49.4) & 0.96 \\ 48.8(20.8-151.7) & 51.8(10-115) & 0.97 \\ 11.6(3.4-268.9) & 12.1(2.3-125.9) & 0.89 \\ 60.9(25.8-266.4) & 66.1(20.5-125.9) & 0.63 \\ 64(25.8-268.9) & 70.9(21.3-126.3) & 0.92\end{array}$


Table 3. Comparisons of demographics, clinical, laboratory and histopathological data and treatment characteristics between patients with sustained remission and relapsers

\begin{tabular}{|c|c|c|c|}
\hline & $\begin{array}{l}\text { Sustained remission } \\
\text { group }(\mathrm{n}=32)\end{array}$ & $\begin{array}{l}\text { Relapse group } \\
\qquad(\mathrm{n}=7)\end{array}$ & $\mathrm{p}$ value \\
\hline Age at diagnosis (years) & $51(16-73)$ & $44(25-64)$ & 0.54 \\
\hline Sex ratio, Females/males & $25 / 7(78 / 22)$ & $4 / 3(57 / 43)$ & 0.31 \\
\hline Other auto-immune diseases & $8(25)$ & $1(14.3)$ & 0.6 \\
\hline Overlap syndrome HAI-CBP & $4(12.5)$ & $0(0.0)$ & 0.39 \\
\hline $\begin{array}{l}\text { Biochemistry at diagnosis } \\
\text { ALT, x ULN, md: } 1 \\
\text { AST, x ULN, md: } 2 \\
\gamma \text {-globulin, x ULN, md: } 23 \\
\text { IgG, x ULN, md: } 22\end{array}$ & $\begin{array}{l}16.3(1.4-81) \\
10.7(1.1-56.3) \\
1.2(0.8-1.9) \\
1.4(0.5-2.1)\end{array}$ & $\begin{array}{l}12.3(4.2-30.7) \\
5.7(2.6-37.1) \\
1.1(0.9-1.2) \\
1.2(1.1-1.6)\end{array}$ & $\begin{array}{l}0.44 \\
0.38 \\
0.75 \\
0.94\end{array}$ \\
\hline $\begin{array}{l}\text { Autoantibodies at diagnosis } \\
\text { ANA } \\
\text { ASMA }\end{array}$ & $\begin{array}{l}25(78) \\
16(50)\end{array}$ & $\begin{array}{l}7(100) \\
7(100)\end{array}$ & $\begin{array}{c}0.18 \\
\mathbf{0 . 0 0 5}\end{array}$ \\
\hline $\begin{array}{l}\text { METAVIR A (Liver biopsy 1) } \\
\text { A0 } \\
\text { A1 } \\
\text { A2 } \\
\text { A3 }\end{array}$ & $\begin{array}{l}0(0.0) \\
5(15.6) \\
9(28.1) \\
18(56.3)\end{array}$ & $\begin{array}{l}0(0.0) \\
1(14.3) \\
2(28.6) \\
4(57.1)\end{array}$ & 0.96 \\
\hline $\begin{array}{l}\text { METAVIR F (Liver biopsy l) } \\
\text { F0F1 } \\
\text { F2 } \\
\text { F3F4 }\end{array}$ & $\begin{array}{l}9(28.1) \\
14(43.8) \\
9(28.1)\end{array}$ & $\begin{array}{l}1(14.3) \\
2(28.6) \\
4(57.1)\end{array}$ & 0.39 \\
\hline $\begin{array}{l}\text { Biochemistry at treatment withdrawal } \\
\text { ALT, x ULN, md: } 1 \\
\text { AST, x ULN, md: } 1 \\
\gamma \text {-globulins, x ULN, md: } 21 \\
\text { IgG, x ULN, md: } 17\end{array}$ & $\begin{array}{l}0.5(0.2-1.0) \\
0.6(0.4-0.9) \\
0.8(0.6-1.0) \\
0.7(0.2-1.0)\end{array}$ & $\begin{array}{l}0.5(0.3-0.6) \\
0.6(0.4-0.8) \\
0.7(0.6-0.7) \\
0.9^{*}\end{array}$ & $\begin{array}{l}0.47 \\
0.62 \\
0.18 \\
-\end{array}$ \\
\hline Liver biopsy 2 & $20(62.5)$ & $4(57)$ & \\
\hline
\end{tabular}


METAVIR A (Liver biopsy 2)

A0

METAVIR F (Liver biopsy 2)

F0F1

F2

F3F4

Treatment

CT monotherapy

CT-AZT bitherapy

Delay to transaminase normalization, $\mathrm{md}: 2^{* *}$

Delay to biochemical remission, $\mathrm{md}: 5^{* *}$

Duration of biochemical remission, md: $5^{* *}$

Duration of CT treatment**

Duration of AZT treatment **

Duration of treatment $* *$

$$
19(95)
$$$$
1(5)
$$

$11(55.0)$

$9(45.0)$

$0(0)$

\section{$4(12.5)$}

$28(87.5)$

$6.2(0.5-44)$

$6.6(1.0-44)$

$51.9(20.3-157.8)$

$13(1.8-268.9)$

$62.7(27.6-266.4)$

66.3 (25.3-268.9)
$2(50)$

$3(75.0)$

$0(0.0)$

$1(25.0)$

$1(14.3)$

6 (85.7)

$6.8(0.8-15.9)$

$6.9(0.9-30.8)$

44 (25.7-74.7)

$16.7(9.2-81.6)$

$44.2(28.3-67)$

$53.3(39.5-81.6)$

Data are expressed as No (\%) unless otherwise indicated. Continuous variables are reported as median (range). ALT: alanine aminotransferase; AST: aspartate aminotransferase; Alk. Phosph.: alkaline phosphatase; ULN: upper limit of normal; ANA: antinuclear antibody; ASMA: anti-smooth muscle antibody; md: missing data; A:activity; F: fibrosis; liver biopsy 1: at diagnosis; liver biospsy 2: at the end of first line treatment; CT: corticosteroid; AZT: azathioprine. * only one dosage was available in this subgroup. ** duration in months 
Table 4. Evolution of fibrosis between liver biopsies performed at AIH diagnosis and at the end of first line treatment in 47 patients with biochemical remission

\begin{tabular}{|c|c|c|c|c|c|c|}
\cline { 3 - 7 } \multicolumn{2}{c|}{} & \multicolumn{5}{c|}{ Liver biopsy at the end of treatment } \\
\cline { 2 - 7 } \multicolumn{1}{c|}{} & $\mathrm{F} 0$ & $\mathrm{~F} 1$ & $\mathrm{~F} 2$ & $\mathrm{~F} 3$ & $\mathrm{~F} 4$ \\
\hline \multirow{4}{*}{$\begin{array}{c}\text { Liver } \\
\text { biopsy at } \\
\text { diagnosis }\end{array}$} & $\mathrm{F} 0$ & 3 & & & & \\
\cline { 2 - 8 } & $\mathrm{F} 1$ & 3 & 5 & 1 & & \\
\cline { 2 - 8 } & $\mathrm{F} 3$ & 4 & 3 & 3 & 3 & \\
\cline { 2 - 8 } & $\mathrm{F} 4$ & 1 & 0 & 5 & 1 & 1 \\
\hline
\end{tabular}


Supplementary Fig. 1. Correlation between serum gammaglobulin and IgG concentrations (23 patients with both dosages on the same day at diagnosis or during follow-up). ULN: upper limit of normal.

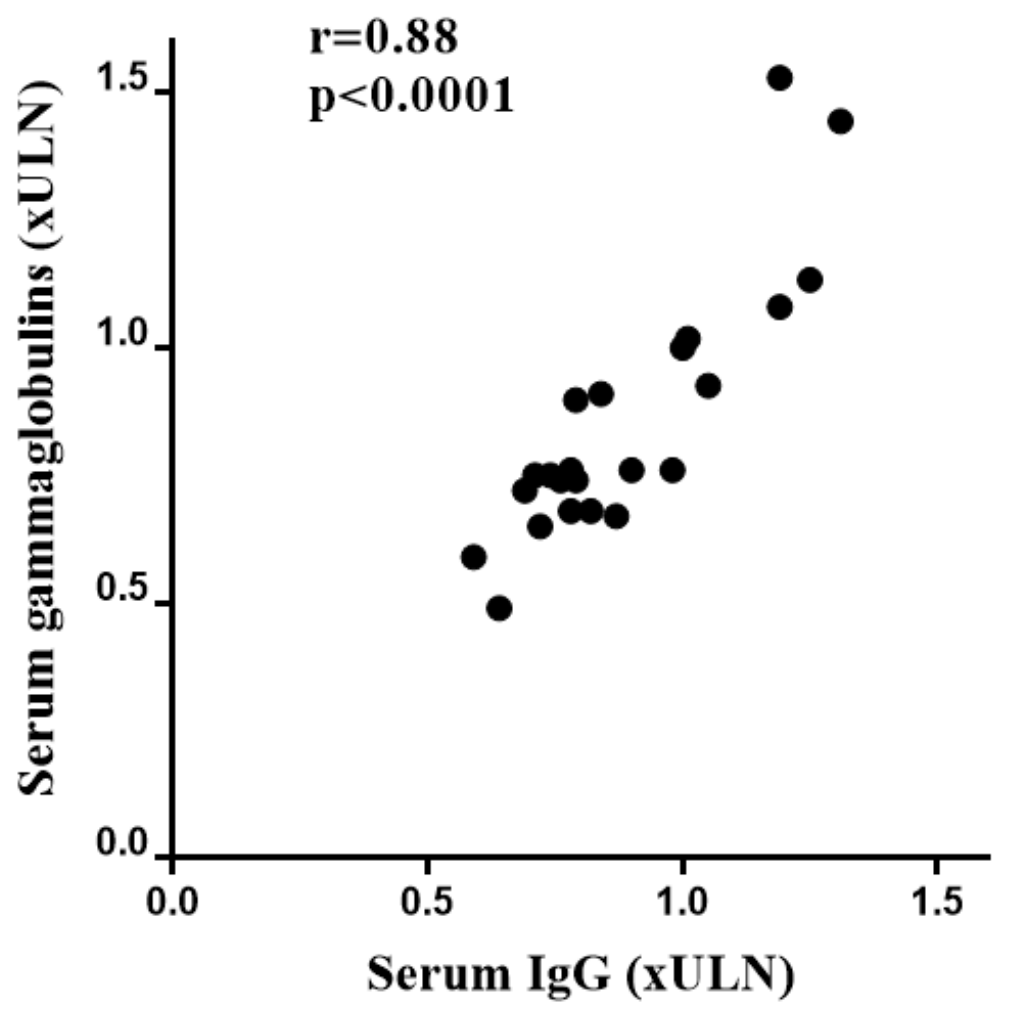


Supplementary Fig.2 Serum gammaglobulin concentration in histological responders and non-responders after a median biochemical response of 48.8 and 51.8 months respectively. A. Median and interquartile range in histological responders and non-responders $(p=0.03$, Wilcoxon test). B. Percentages of histological responders in patients with gammaglobulins < $0.8 \times$ ULN and those with gammaglobulins in the normal range but above $0.8 \times$ ULN (p $=0.049$, Fisher's exact test). ULN: upper level of normal. Missing data $=22$

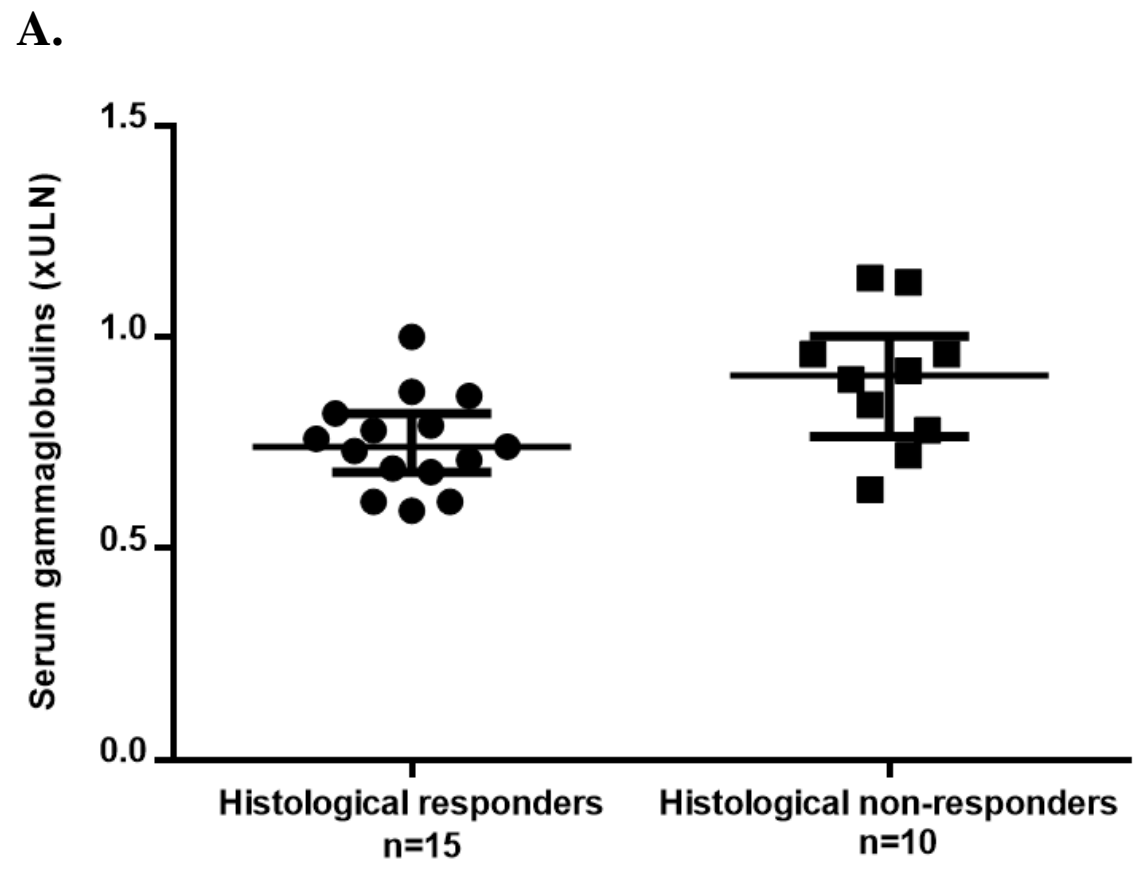

B.

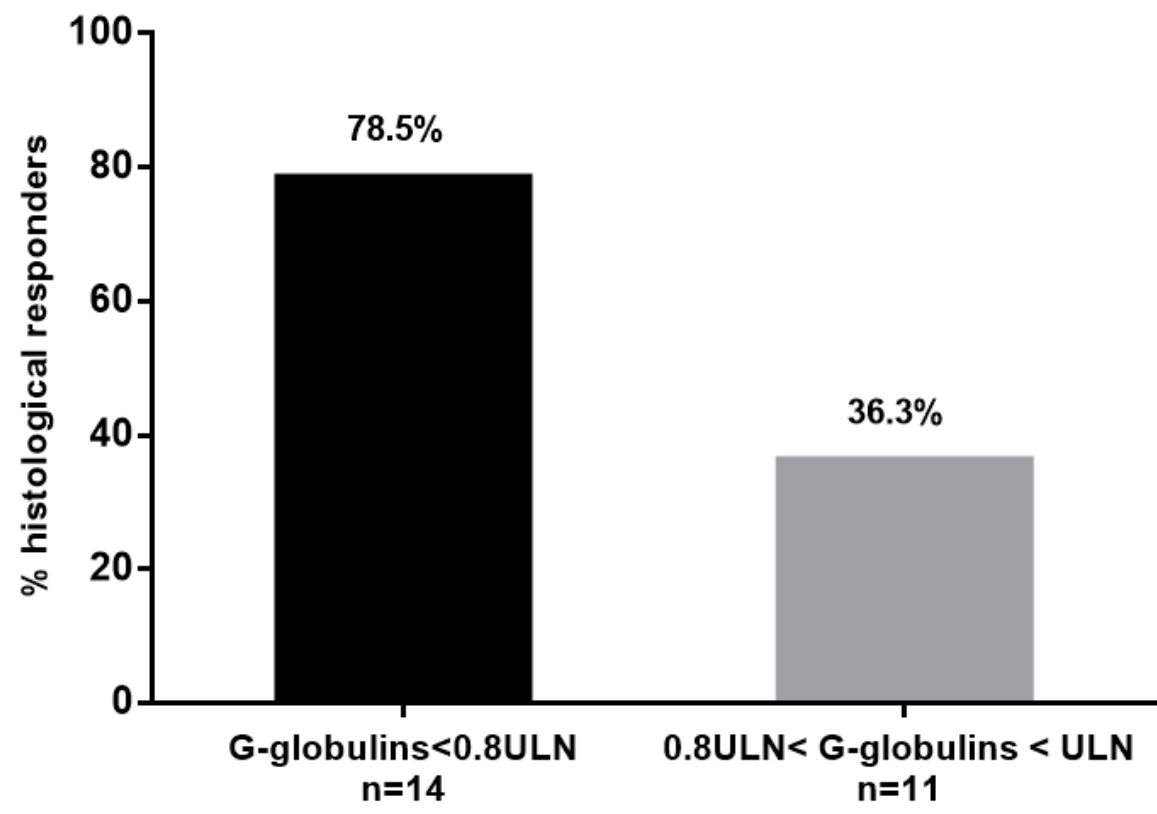


Supplementary Fig. 3. Kaplan Meier plot: Probability of relapse in 35 AIH patients with prolonged biochemical remission (3 patients with drug-induced AIH and1 patient with AIH triggered by cytomegalovirus infection have been excluded). The cumulative rate of relapse was $28.9 \%$ at 64 months. The median time of relapse was 9.4 months (range1.5-64).

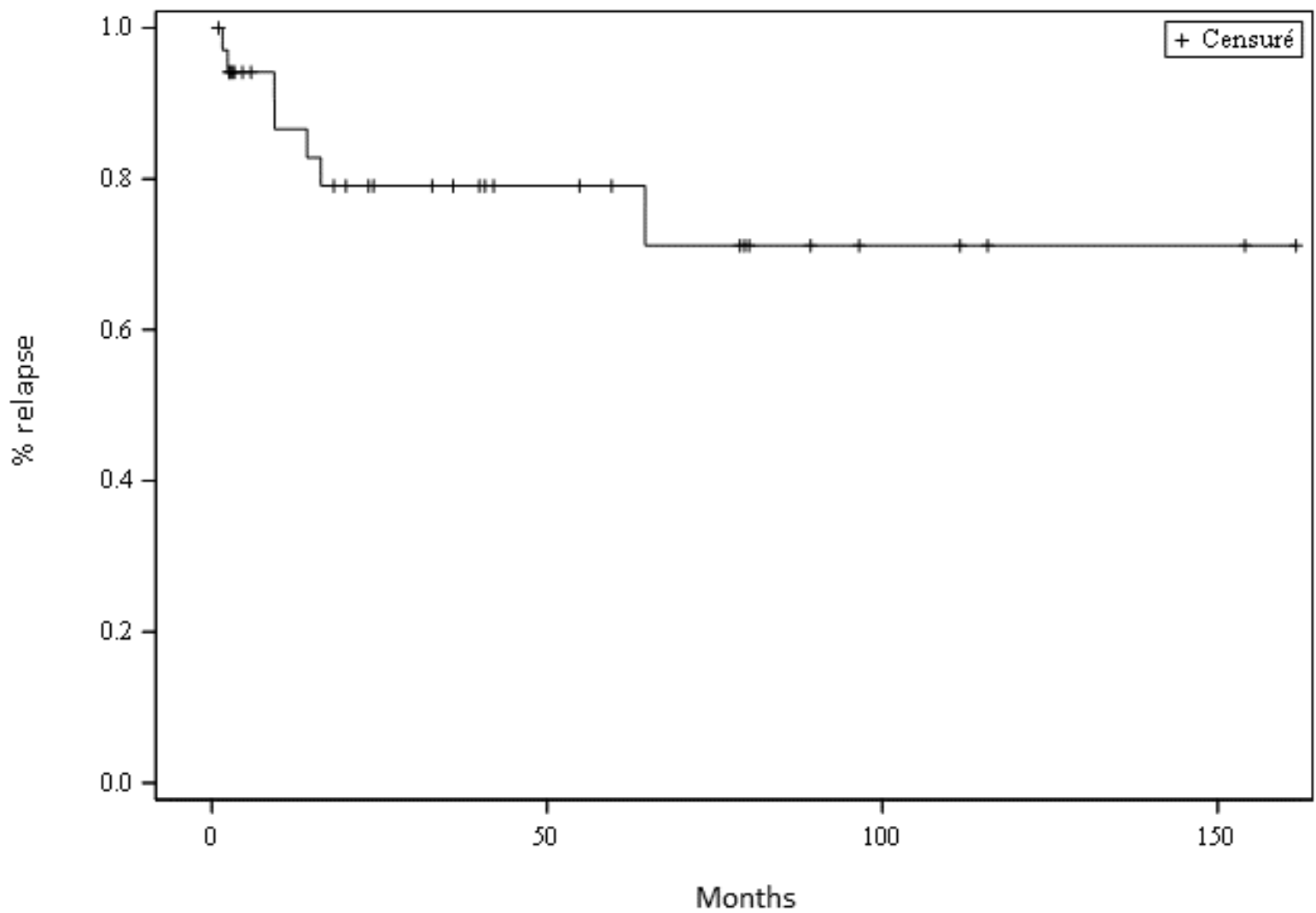

